\title{
Mechanical power normalized to lung-thorax compliance predicts prolonged ventilation weaning failure: a prospective study
}

\author{
Alessandro Ghiani ${ }^{* *} \mathbb{D}$, Joanna Paderewska', Swenja Walcher ${ }^{1}$ and Claus Neurohr ${ }^{1,2}$
}

\begin{abstract}
Background: Mechanical power (MP) of artificial ventilation, the energy transferred to the respiratory system, is a chief determinant of adequate oxygenation and decarboxylation. Calculated MP, the product of applied airway pressure and minute ventilation, may serve as an estimate of respiratory muscle workload when switching to spontaneous breathing. The aim of the study was to assess MP's discriminatory performance in predicting successful weaning from prolonged tracheostomy ventilation.

Methods: Prospective, observational study in 130 prolonged mechanically ventilated, tracheotomized patients in a specialized weaning center. Predictive weaning outcome ability of arterial blood gas analyses and indices derived from calculated MP at beginning and end of weaning was determined in terms of area under receiver operating characteristic curve (AUROC) and measures derived from k-fold cross-validation (likelihood ratios, diagnostic odds ratio, $\mathrm{F}_{1}$ score, and Matthews correlation coefficient [MCC]).
\end{abstract}

Results: Forty-four (33.8\%) patients experienced weaning failure. Absolute MP showed poor discrimination in predicting outcome; whereas specific MP (MP normalized to dynamic lung-thorax compliance, LTC $_{\text {dyn }}$-MP) had moderate diagnostic accuracy (MCC 0.38; AUROC 0.79, 95\%Cl [0.71-0.86], $\mathrm{p}<0.001$ ), further improved by correction for corresponding mechanical ventilation $\mathrm{P}_{\mathrm{a}} \mathrm{CO}_{2}$ (termed the power index of the respiratory system $[\mathrm{PI}$ rs]: MCC 0.52; AUROC 0.86 [0.79-0.92], $\mathrm{p}<0.001$ ). Diagnostic performance of MP indices increased over the course of weaning, with maximum accuracy immediately before completion ( $\mathrm{LTC}_{\mathrm{dyn}}$-MP: MCC 0.49; AUROC 0.86 [0.78-0.91], p<0.001; $\mathrm{Pl}_{\mathrm{rs}}$ : MCC 0.68; AUROC 0.92 [0.86-0.96], $\mathrm{p}<0.001)$.

Conclusions: MP normalized to dynamic lung-thorax compliance, a surrogate for applied power per unit of ventilated lung volume, accurately discriminated between low and high risk for weaning failure following prolonged mechanical ventilation.

Keywords: Mechanical ventilation, Ventilator weaning, Tracheostomy

*Correspondence: alessandro.ghiani@klinik-schillerhoehe.de ${ }^{1}$ Department of Pulmonology and Respiratory Medicine, Schillerhoehe Lung Clinic (affiliated to the Robert-Bosch-Hospital GmbH, Stuttgart), Solitudestrasse 18, 70839 Gerlingen, Germany

This work was performed at the Schillerhoehe Lung Clinic, Gerlingen (Germany)

Full list of author information is available at the end of the article

\begin{abstract}
Background
Weaning from prolonged tracheostomy ventilation is often seen as an art rather than a scientifically based, standardized process. The main reason may be that these patients have so far attracted little attention from scientists, resulting in a lack of evidence-based recommendations, with significant differences in approaches at dedicated weaning and home ventilation centers [1-3].
\end{abstract}


Furthermore, predictors of weaning and extubation outcome have so far been predominantly validated in patients following short-term mechanical ventilation [4]. Diagnostic accuracy of such predictors (e.g. maximum inspiratory pressure, minute ventilation, respiratory rate) varies considerably, perhaps due to limited agreement between these parameters and the underlying pathophysiology of weaning failure, in prolonged ventilated patients namely an imbalance between work of breathing and capacity of respiratory muscles $[5,6]$. The mechanical power (MP) of artificial ventilation, the energy transferred to the respiratory system per time unit, is a chief determinant for the establishment of adequate gas exchange. Calculated MP, the product of the applied airway pressure and minute ventilation, may therefore serve as an estimate for the workload imposed on respiratory muscles when switching to spontaneous breathing, which is a crucial factor when evaluating patients' ability to wean from mechanical ventilation. In a recent study, MP normalized to dynamic lung thorax compliance, assessed immediately before the first spontaneous breathing trial (SBT) upon admission to the weaning center, was independently associated with failure of prolonged weaning [7].

The aim of the present study was to assess the diagnostic accuracy of MP in predicting the outcome of prolonged weaning in tracheotomized patients, treated at a specialized weaning center.

\section{Methods}

This was a prospective, observational study conducted at a national weaning center in Germany. The study was approved by the local institutional review board for human studies (Ethics Committee of the State Chamber of Physicians of Baden-Wuerttemberg, Germany, file number F-2018-116), and written informed consent was obtained from all patients or a legal representative.

\section{Patient selection}

Patients were included in the analyses if they were referred because of evident failure to wean from tracheostomy ventilation, and if they met the criteria of prolonged weaning, classified as Category 3 as defined by Boles and colleagues [8]. Exclusion criteria were a confirmed diagnosis of neuromuscular disease, death prior to weaning completion, and declined consent for participation.

\section{Ventilator weaning}

Weaning was systematically performed according to the recommendations of Boles and colleagues [8], and the national guidelines on prolonged weaning [9], including protocol-based increasing periods of unassisted breathing through a tracheostomy collar (weaning trials) [10]. In the intervals between SBT, all patients were ventilated in the pressure-controlled, assisted-controlled (A/C) mode (Vivo 50/55, Breas Medical AB, Moelnlycke, Sweden) to recover from the imposed work of breathing during SBT (further details can be found in the online supplement).

\section{Variables analyzed to predict weaning outcome}

Ventilator variables and the corresponding arterial blood gas analysis were collected $48 \mathrm{~h}$ after the first SBT upon admission to the weaning center and $48 \mathrm{~h}$ before weaning completion, with the median of these values used for the analyses (Fig. 1).

The following parameters were calculated from the collected variables: dynamic lung-thorax compliance $\left(\operatorname{LTC}_{\mathrm{dyn}}\right)$ [11], mechanical power [12, 13], and ventilatory ratio (VR) [14]. Specific MP indices were calculated by normalizing absolute values to predicted body weight (a surrogate of the total lung capacity, PBW-MP) [15], and to $\mathrm{LTC}_{\mathrm{dyn}}$ (reflecting actual ventilated lung volume, $\mathrm{LTC}_{\mathrm{dyn}}-\mathrm{MP}$ ) [7]. Finally, $\mathrm{LTC}_{\mathrm{dyn}}-\mathrm{MP}$ was corrected for the corresponding mechanical ventilation $\mathrm{P}_{\mathrm{a}} \mathrm{CO}_{2}$ [7]

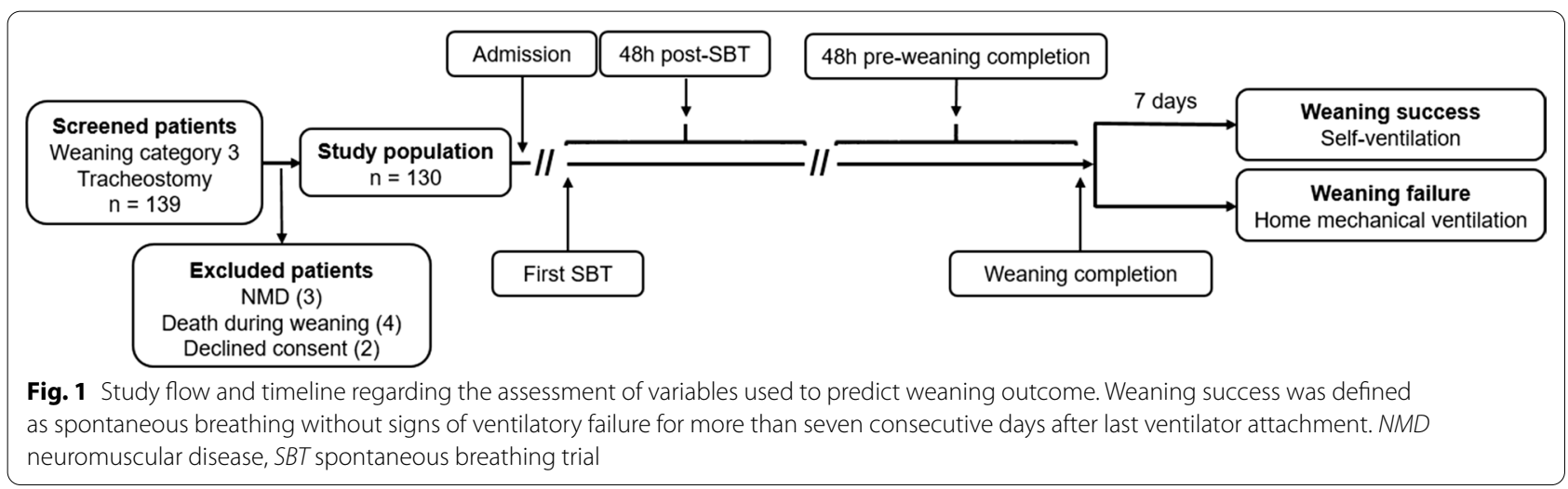


(simulating isocapnic conditions), termed the "power index of the respiratory system" $\left(\mathrm{PI}_{\mathrm{rs}}\right)$, a surrogate of applied specific power necessary to provide adequate decarboxylation (further details can be found in the online supplement).

\section{Classification of outcomes}

Patients were classified at the end of ventilator weaning into two categories: patients with weaning success, and those with weaning failure.

Successful weaning was defined as spontaneous breathing for more than seven consecutive days without concomitant clinical or laboratory signs of chronic ventilatory insufficiency after weaning completion. Weaning completion was determined by the last day on which the patient was attached to the ventilator. Weaning failure referred to a state of chronic ventilatory insufficiency in the course of weaning, defined as recurrent hypercapnia during daily weaning trials, preventing the extension of spontaneous respiration $\left(\mathrm{P}_{\mathrm{a}} \mathrm{CO}_{2}>45.0 \mathrm{mmHg}\right.$ on at least two occasions), or hypercapnia occurring during spontaneous breathing within seven consecutive days after weaning completion [7] (median $\mathrm{P}_{\mathrm{a}} \mathrm{CO}_{2}>45.0 \mathrm{mmHg}$ derived from the highest measured $\mathrm{P}_{\mathrm{a}} \mathrm{CO}_{2}$ on each of the seven days), usually requiring resumption of ventilation with transition to home mechanical ventilation (either by face mask or by tracheostomy tube).

Based on these criteria, the decision to terminate the weaning process was finally made by an interdisciplinary team including the attending physician and the respiratory therapist. Domiciliary ventilation was prescribed for at least $6 \mathrm{~h}$ per day [16], with the duration adjusted to patients' need, aiming at sufficiently unloading respiratory muscles, indicated by normocapnia during both mechanical and self-ventilation.

\section{Sample size determination and statistical analysis}

Descriptive and frequency statistics were used to summarize patients' demographics and baseline characteristics. Differences between groups in categorical variables were analyzed using Chi-square or Fisher's exact tests, as appropriate. Continuous variables were subjected to Kolmogorov-Smirnov normality test for homogeneity of variance, and, depending on statistical distribution, either Student's $t$-test or Mann-Whitney $U$-test was used to examine differences in these parameters.

To assess the ability of the variables to predict weaning outcome, we performed a receiver operating characteristic (ROC) curve analysis in the entire study population with diagnostic performance expressed as area under the ROC curve (AUROC). We also performed a prospective, stratified, 2-times repeated, twofold cross-validation [17] (see Additional file 1: Figure S1, Figure S2, Table S1), with the resulting performance of each index derived from the test sets expressed as sensitivity, specificity, positive/negative predictive value, accuracy, positive/negative likelihood ratio, diagnostic odds ratio (DOR), $\mathrm{F}_{1}$ score, and Matthews correlation coefficient (MCC).

Pearson's $r$ was used for assessment of correlation between the index tests and daily duration of mechanical ventilation following (unsuccessful) weaning completion.

Based on an $\alpha$-level (type-I error) of 0.05 with power $(1-\beta)$ of $80 \%$ and prevalence of weaning failure (as defined above) of $40 \%$ [7], we calculated a sample size of 124 patients to capture variables with at least moderate diagnostic accuracy (AUROC $>0.70$ ) from the training sets. All tests were two-tailed; statistical significance was indicated by $p<0.05$. All analyses were performed using MedCalc statistical software version 19.2.5 (MedCalc Software Ltd, Ostend, Belgium). Further details on the statistical methods can be found in the online supplement.

\section{Results}

Of 139 consecutive patients screened for eligibility between March 2019 and August 2020, 130 (93.5\%) were included in the analyses. Reasons for exclusion were a confirmed diagnosis of neuromuscular disease in three patients, four patients died during weaning, and two patients declined consent.

Clinical characteristics differed between patients with successful and unsuccessful weaning mainly in terms of smoking history, presence of chronic obstructive pulmonary disease (COPD) as a comorbidity, and acute exacerbation of COPD as the reason for respiratory failure (Table 1).

Weaning failure occurred in 44 patients (33.8\%), 42 of whom remained ventilated on hospital discharge (19 on non-invasive home mechanical ventilation and 23 discharged with permanent tracheostomy ventilation) (see Additional file 1: Table S2).

Patients' baseline clinical characteristics and outcomes were equally distributed after each randomization to one of the two groups (see Additional file 1: Table S1). Table 2 shows the mean threshold values derived from the training sets that best predicted weaning failure.

A good diagnostic performance in predicting failure of prolonged weaning from values collected $48 \mathrm{~h}$ after the first SBT was shown for $\mathrm{P}_{\mathrm{a}} \mathrm{CO}_{2}$ on mechanical ventilation (DOR 11.1, MCC 0.50, AUROC 0.81 [0.73-0.87]), and the PI $\mathrm{r}_{\mathrm{rs}}$ (DOR 15.3, MCC 0.52, AUROC 0.86 [0.79-0.92]) (Tables 3 and 4).

An excellent performance in predicting unsuccessful weaning from values assessed $48 \mathrm{~h}$ before weaning completion was observed for $\mathrm{LTC}_{\mathrm{dyn}}$-MP (DOR 18.5, MCC 0.49 , AUROC $0.86[0.78-0.91]$ ) and especially for $\mathrm{PI}_{\mathrm{rs}}$ 
Table 1 Clinical characteristics on admission to the weaning center- comparison of patients with weaning success and weaning failure

\begin{tabular}{|c|c|c|c|c|}
\hline Clinical characteristics & $\begin{array}{l}\text { All patients } \\
(n=130)\end{array}$ & $\begin{array}{l}\text { Weaning success } \\
(n=86)\end{array}$ & $\begin{array}{l}\text { Weaning failure } \\
(n=44)\end{array}$ & $P$ value ${ }^{a}$ \\
\hline Age (years) & $69(60-76)$ & $68(59-76)$ & $70(63-75)$ & $0.609^{c}$ \\
\hline Gender (male) & $82(63.1)$ & $59(68.8)$ & $23(52.3)$ & $0.069^{d}$ \\
\hline Body mass index $\left(\mathrm{kg} / \mathrm{m}^{2}\right)$ & $27.9( \pm 6.9)$ & $27.3( \pm 6.6)$ & $29.1( \pm 7.4)$ & $0.369^{c}$ \\
\hline Obesity $\left(\mathrm{BMI} \geq 30 \mathrm{~kg} / \mathrm{m}^{2}\right)$ & $40(30.8)$ & $24(27.9)$ & $16(36.4)$ & $0.325^{d}$ \\
\hline Smoking history & $48(36.9)$ & $21(24.5)$ & $27(61.4)$ & $<0.001^{d}$ \\
\hline APACHE-II (points) & $15.9( \pm 5.3)$ & $15.8( \pm 5.3)$ & $15.9( \pm 5.4)$ & $0.905^{\mathrm{b}}$ \\
\hline Albumin (g/dL) & $2.2( \pm 0.5)$ & $2.2( \pm 0.6)$ & $2.2( \pm 0.5)$ & $0.338^{c}$ \\
\hline Ventilator days on admission & $25(16-34)$ & $27(17-35)$ & $23(16-30)$ & $0.123^{c}$ \\
\hline Time from Intubation to tracheostomy (days) & $12(7-18)$ & $13(7-19)$ & $10(6-15)$ & $0.057^{c}$ \\
\hline ECLA & $14(10.8)$ & $13(15.1)$ & $1(2.3)$ & $0.026^{d}$ \\
\hline \multicolumn{5}{|l|}{ Reason for mechanical ventilation } \\
\hline Pneumonia & $51(39.2)$ & $31(36.0)$ & $20(45.5)$ & $0.300^{d}$ \\
\hline SARS-CoV-2 infection & $4(3.1)$ & $4(4.7)$ & $0(0.0)$ & - \\
\hline Surgery & $32(24.6)$ & $25(29.1)$ & $7(15.9)$ & $0.101^{d}$ \\
\hline Cardiopulmonary resuscitation & $10(7.7)$ & $8(9.3)$ & $2(4.5)$ & $0.337^{d}$ \\
\hline Acute exacerbation of COPD & $10(7.7)$ & $2(2.3)$ & $8(18.2)$ & $0.001^{d}$ \\
\hline Acute heart failure & $6(4.6)$ & $5(5.8)$ & $1(2.3)$ & $0.663^{e}$ \\
\hline Sepsis (including septic shock) & $7(5.4)$ & $5(5.8)$ & $2(4.5)$ & $1.000^{\mathrm{e}}$ \\
\hline Other & $17(13.1)$ & $10(11.6)$ & $7(15.9)$ & $0.495^{\mathrm{d}}$ \\
\hline \multicolumn{5}{|l|}{ Comorbidities } \\
\hline Charlson comorbidity index (points) & $5.5( \pm 2.3)$ & $5.4( \pm 2.4)$ & $5.6( \pm 2.1)$ & $0.611^{c}$ \\
\hline Renal insufficiency & $46(35.4)$ & 36(41.9) & $10(22.7)$ & $0.032^{d}$ \\
\hline Hemodialysis & $24(18.5)$ & $21(24.4)$ & $3(6.8)$ & $0.289^{d}$ \\
\hline Diabetes mellitus & $35(26.9)$ & $20(23.3)$ & $15(34.1)$ & $0.189^{d}$ \\
\hline Coronary artery disease & $33(25.4)$ & $23(26.7)$ & $10(22.7)$ & $0.620^{d}$ \\
\hline COPD & $30(23.1)$ & $5(5.8)$ & $25(56.8)$ & $<0.001^{d}$ \\
\hline Chronic heart failure & $17(13.1)$ & $14(16.3)$ & $3(6.8)$ & $0.132^{d}$ \\
\hline Malignancy & $10(7.7)$ & $7(8.1)$ & $3(6.8)$ & $0.790^{\mathrm{d}}$ \\
\hline Hepatopathy & $7(5.4)$ & $5(5.8)$ & $2(4.5)$ & $1.000^{\mathrm{e}}$ \\
\hline Interstitial lung disease & $8(6.2)$ & $6(7.0)$ & $2(4.5)$ & $0.716^{e}$ \\
\hline
\end{tabular}

Continuous variables are presented as arithmetic means ( \pm standard deviation) or median (- interquartile range [IQR]); categorical variables are presented as number (\%)

$B M I$ body mass index, APACHE-II Acute Physiology and Chronic Health Evaluation II score, ECLA extracorporeal lung assistance (in acute respiratory failure), SARS-CoV-2 severe acute respiratory syndrome-Coronavirus 2, COPD chronic obstructive pulmonary disease

${ }^{\text {a }} P$ value for differences between patients with weaning success and weaning failure

b Student's $t$-test

' Mann-Whitney U-test

${ }^{d}$ Chi-squared test

${ }^{\mathrm{e}}$ Fisher's exact test

(sensitivity $88 \%$, specificity $83 \%$, DOR 52.8 MCC 0.68 , AUROC 0.92 [0.86-0.96]). In contrast, absolute mechanical power had moderate diagnostic accuracy for both timepoints, whereas $\mathrm{PI}_{\mathrm{rs}}$ predicted weaning failure significantly better than MP or $\mathrm{LTC}_{\mathrm{dyn}}$-MP (Fig. 2).

In the 62 patients with median $\mathrm{PI}_{\mathrm{rs}}^{2.0}<3704 \mathrm{cmH}_{2} \mathrm{O}^{2}$ / min (corresponding to the mean threshold $48 \mathrm{~h}$ before weaning completion derived from the training sets, Table 2) at both the beginning and the end of weaning, only three patients $(4.8 \%)$ remained ventilator-dependent on discharge.

Since the diagnostic accuracy of an index derived from cross-validation largely depends on the selected threshold, a second analysis was performed using the criteria associated with the Youden index, resulting 
Table 2 Threshold values of variables used to predict weaning outcome-mean values derived from the training sets

\begin{tabular}{lll}
\hline Variables & Failure of prolonged weaning \\
\cline { 2 - 3 } & Post-SBT & $\begin{array}{l}\text { Pre- } \\
\text { weaning } \\
\text { completion }\end{array}$ \\
\hline $\mathrm{P}_{\mathrm{a}} \mathrm{CO}_{2}$ on $\mathrm{MV}(\mathrm{mmHg})$ & $>35.3$ & $>34.6$ \\
$\mathrm{VR}$ & $>1.23$ & $>1.23$ \\
$\mathrm{LTC}$ & $\leq 31.2$ & $\leq 33.6$ \\
$\mathrm{MP}(\mathrm{J} / \mathrm{min})$ & $>20.8$ & $>20.3$ \\
$\mathrm{PBW}-\mathrm{MP}(\mathrm{J} / \mathrm{min} / \mathrm{kg})$ & $>0.3164$ & $>0.3158$ \\
$\mathrm{LTC}_{\mathrm{dyn}}-\mathrm{MP}\left(\mathrm{cmH}_{2} \mathrm{O}^{2} / \mathrm{min}\right)$ & $>6496$ & $>6341$ \\
$\mathrm{PI}_{\mathrm{rs}}{ }^{1.0}\left(\mathrm{~cm} \mathrm{H}_{2} \mathrm{O}^{2} / \mathrm{min}^{2}\right)$ & $>5020$ & $>4915$ \\
$\mathrm{Pl}_{\mathrm{rs}}{ }^{2.0}\left(\mathrm{~cm} \mathrm{H}_{2} \mathrm{O}^{2} / \mathrm{min}\right)$ & $>3895$ & $>3704$
\end{tabular}

$>/ \leq$ indicate whether values above/below the threshold value predicted failure of prolonged weaning. The associated criterion is the threshold value that minimized the difference between sensitivity and specificity of the test, graphically corresponding to the intersection of the line connecting the leftupper corner and the right-lower corner of the unit square and the ROC curve $S B T$ spontaneous breathing trial, $M V$ mechanical ventilation, $V R$ ventilatory ratio, $L T C_{d y n}$ dynamic lung-thorax compliance, MP mechanical power, $P B W-M P$ mechanical power normalized to predicted body weight, $L T C_{d y n}-M P$ mechanical power normalized to dynamic lung-thorax compliance, $P I_{r s}$ power index of the respiratory system in similar diagnostic performance of variables derived from mechanical power (see Additional file 1: Table S3).

When comparing the discriminatory performance of PBW-MP and $\mathrm{LTC}_{\mathrm{dyn}}-\mathrm{MP}$, both of which are indices relating transferred power to surrogates of lung volume, LTC $_{\mathrm{dyn}}$-MP consistently performed better in predicting weaning outcome (Table 4). These differences in AUROC reached statistical significance $48 \mathrm{~h}$ before weaning completion (0.75 [0.66-0.82] vs 0.86 [0.78-0.91], p=0.004).

Comparison of the diagnostic performance of $\mathrm{PI}_{\mathrm{rs}}^{2.0}$ from patients with less and more than $50 \%$ of variables recorded during pressure-assisted ventilation revealed neither a significant difference in predicting weaning outcome from values collected $48 \mathrm{~h}$ after the first SBT (AUROC 0.92 [0.83-0.98] vs 0.80 [0.69-0.89], $\mathrm{p}=0.056$ ), nor from values assessed $48 \mathrm{~h}$ before weaning completion (0.95 [0.85-0.99] vs 0.91 [0.83-0.96], $\mathrm{p}=0.423)$.

Finally, Pearson's $r$ showed a significant correlation of $\mathrm{LTC}_{\text {dyn }}-\mathrm{MP}(r=0.44,95 \% \mathrm{CI} 0.29-0.57 ; \mathrm{p}<0.001)$ and $\mathrm{PI}_{\mathrm{rs}}$ $(0.61$ [0.49-0.71]; $\mathrm{p}<0.001)$ at end of weaning with the duration of daily home ventilation $(12.0 \pm 7.9 \mathrm{~h}$ in weaning failure patients).

Further details on differences in ventilator variables and mechanical power indices between the successful and

Table 3 Cross-validated performance of variables analyzed to predict weaning outcome — mean values derived from the test sets

\begin{tabular}{|c|c|c|c|c|c|c|c|c|c|c|}
\hline \multirow[t]{2}{*}{ Variables } & \multicolumn{10}{|c|}{ Failure of prolonged weaning } \\
\hline & Sens & Spec & PPV & NPV & Accuracy & PLR & NLR & DOR & $F_{1}$ & MCC \\
\hline \multicolumn{11}{|l|}{ Post-SBT } \\
\hline $\mathrm{P}_{\mathrm{a}} \mathrm{CO}_{2}$ on $\mathrm{MV}$ & $78(56-93)$ & $73(58-85)$ & $61(47-73)$ & $87(75-94)$ & $75(63-85)$ & $3.1(1.7-5.5)$ & $0.3(0.7-0.1)$ & 11.1 & 0.68 & 0.50 \\
\hline VR & $73(50-89)$ & $71(55-84)$ & $56(43-69)$ & $84(72-91)$ & $72(59-82)$ & $2.6(1.5-4.4)$ & $0.4(0.8-0.2)$ & 7.2 & 0.63 & 0.42 \\
\hline $\mathrm{LTC}_{\text {dyn }}$ & $68(46-85)$ & $71(55-84)$ & $54(40-67)$ & $82(71-89)$ & $70(57-81)$ & $2.4(1.4-4.1)$ & $0.4(0.8-0.3)$ & 7.7 & 0.60 & 0.38 \\
\hline MP & $66(44-84)$ & $64(48-78)$ & $48(36-53)$ & $79(67-87)$ & $65(52-76)$ & $1.8(1.1-3.0)$ & $0.5(1.0-0.3)$ & 4.5 & 0.55 & 0.28 \\
\hline PBW-MP & $68(45-86)$ & $68(52-81)$ & $53(40-66)$ & 81 (69-89) & $68(55-79)$ & $2.3(1.3-4.1)$ & $0.5(0.9-0.3)$ & 5.6 & 0.59 & 0.35 \\
\hline $\mathrm{LTC}_{\text {dyn }}-\mathrm{MP}$ & $73(51-88)$ & $67(54-80)$ & $53(41-65)$ & $83(71-90)$ & $69(56-80)$ & $2.3(1.4-3.9)$ & $0.4(0.8-0.2)$ & 7.3 & 0.61 & 0.38 \\
\hline $\mathrm{PI}_{\mathrm{rs}} 1.0$ & $74(52-89)$ & $73(58-86)$ & $58(45-71)$ & $85(73-92)$ & $73(61-84)$ & $2.9(1.6-4.1)$ & $0.4(0.7-0.2)$ & 11.3 & 0.65 & 0.45 \\
\hline $\mathrm{PI}_{\mathrm{rs}}^{2.0}$ & $78(56-92)$ & $76(61-88)$ & $63(49-75)$ & 87 (75-94) & 77 (65-86) & $3.4(1.9-6.2)$ & $0.3(0.6-0.1)$ & 15.3 & 0.70 & 0.52 \\
\hline \multicolumn{11}{|c|}{ Pre-weaning completion } \\
\hline $\mathrm{P}_{\mathrm{a}} \mathrm{CO}_{2}$ on $\mathrm{MV}$ & $77(55-92)$ & $76(60-87)$ & $62(48-74)$ & $87(75-93)$ & $76(64-86)$ & $3.2(1.8-5.8)$ & $0.3(0.7-0.1)$ & 11.6 & 0.69 & 0.51 \\
\hline VR & $70(47-88)$ & $66(50-79)$ & $52(39-64)$ & $81(69-90)$ & $67(55-78)$ & $2.1(1.3-3.5)$ & $0.5(0.9-0.2)$ & 4.8 & 0.59 & 0.35 \\
\hline $\mathrm{LTC}_{\text {dyn }}$ & $69(44-88)$ & $76(61-87)$ & $56(41-69)$ & $86(75-92)$ & $74(62-84)$ & $3.2(1.7-6.3)$ & $0.4(0.8-0.2)$ & 9.4 & 0.61 & 0.43 \\
\hline MP & $70(48-87)$ & $60(44-74)$ & $48(37-59)$ & $80(67-89)$ & $63(51-75)$ & $1.8(1.1-2.9)$ & $0.5(1.0-0.2)$ & 3.8 & 0.57 & 0.29 \\
\hline PBW-MP & $68(45-86)$ & $67(51-80)$ & $51(39-64)$ & $81(68-89)$ & $67(55-78)$ & $2.1(1.2-3.5)$ & $0.5(0.9-0.3)$ & 4.6 & 0.58 & 0.33 \\
\hline $\mathrm{LTC}_{\text {dyn }}-\mathrm{MP}$ & $74(53-88)$ & 77 (62-89) & $62(47-75)$ & $86(75-92)$ & $76(64-86)$ & $3.3(1.8-6.2)$ & $0.3(0.7-0.2)$ & 18.5 & 0.67 & 0.49 \\
\hline $\mathrm{PI}_{\mathrm{rs}}{ }^{1.0}$ & $82(61-94)$ & $81(66-91)$ & $68(53-81)$ & $90(78-95)$ & $81(70-90)$ & $4.4(2.3-8.5)$ & $0.2(0.6-0.1)$ & 42.9 & 0.74 & 0.61 \\
\hline $\mathrm{PI}_{\mathrm{rs}}^{2.0}$ & 88 (67-97) & 83 (69-93) & $73(58-84)$ & $93(81-97)$ & $85(74-92)$ & $5.9(2.8-13)$ & $0.2(0.5-0.1)$ & 52.8 & 0.79 & 0.68 \\
\hline
\end{tabular}

Assessment of mean sensitivity and specificity, positive and negative predictive value, positive and negative likelihood ratio, diagnostic odds ratio, $\mathrm{F}_{1}$ score, and Matthews correlation coefficient (with 95\% confidence intervals)

$S B T$ spontaneous breathing trial, Sens sensitivity, Spec specificity, $P P V$ positive predictive value, NPV negative predictive value, $P L R$ positive likelihood ratio, NLR negative likelihood ratio, $D O R$ diagnostic odds ratio, $F_{1} \mathrm{~F}_{1}$ score, $M C C$ Matthews correlation coefficient, $M V$ mechanical ventilation, $V R$ ventilatory ratio, $L T C_{d y n}$ dynamic lung-thorax compliance, $M P$ mechanical power, $P B W-M P$ mechanical power normalized to predicted body weight, $L T C_{d y n}-M P$ mechanical power normalized to dynamic lung-thorax compliance, $P I_{r s}$ power index of the respiratory system 
Table 4 Area under the ROC curve for each variable-all patients

\begin{tabular}{lll}
\hline Variables & \multicolumn{2}{l}{ Failure of prolonged weaning } \\
\cline { 2 - 3 } & Post-SBT & Pre-weaning completion \\
\hline $\mathrm{P}_{\mathrm{a}} \mathrm{CO}_{2}$ on MV & $0.81(0.73-0.87)$ & $0.86(0.78-0.91)$ \\
$\mathrm{VR}$ & $0.78(0.70-0.85)$ & $0.74(0.66-0.81)$ \\
$\mathrm{LTC}_{\text {dyn }}$ & $0.74(0.65-0.81)$ & $0.82(0.75-0.89)$ \\
$\mathrm{MP}$ & $0.74(0.65-0.81)$ & $0.70(0.61-0.77)$ \\
$\mathrm{PBW}-\mathrm{MP}$ & $0.76(0.68-0.83)$ & $0.75(0.66-0.82)$ \\
$\mathrm{LTC}_{\text {dyn }}-\mathrm{MP}$ & $0.79(0.71-0.86)$ & $0.86(0.78-0.91)$ \\
$\mathrm{PI}_{\mathrm{rs}}{ }^{1.0}$ & $0.84(0.76-0.90)$ & $0.91(0.84-0.95)$ \\
$\mathrm{PI}_{\mathrm{rs}}{ }^{2.0}$ & $0.86(0.79-0.92)$ & $0.92(0.86-0.96)$ \\
\hline
\end{tabular}

The accuracy of each variable in the whole study population presented as the area under the ROC curve with $95 \%$ confidence intervals

$S B T$ spontaneous breathing trial, $M V$ mechanical ventilation, $V R$ ventilatory ratio, $L T C_{d y n}$ dynamic lung-thorax compliance, $M P$ mechanical power, $P B W-M P$ mechanical power normalized to predicted body weight, $L T C_{d y n}-M P$ mechanical power normalized to dynamic lung-thorax compliance, $P I_{r s}$ power index of the respiratory system

unsuccessful weaning group (Additional file 1: Table S4), and ROC curves of selected variables (Additional file 1: Figure S3) can be found in the online data supplement (see Additional file 1).

\section{Discussion}

The aim of the present study was to determine the diagnostic accuracy of MP in predicting successful weaning from prolonged tracheostomy ventilation. Absolute MP showed poor discriminatory performance, whereas specific MP (MP normalized to surrogates of lung dimension, including well-inflated lung tissue determined by computed tomography analysis [18], functional residual capacity, PBW or $\mathrm{LTC}_{\mathrm{dyn}}$ ) had good diagnostic accuracy, further improved by correction for the corresponding $\mathrm{P}_{\mathrm{a}} \mathrm{CO}_{2}$ on mechanical ventilation. Moreover, discriminatory performance of the MP indices increased over the course of weaning, with maximum accuracy observed immediately before weaning completion.

The concept of MP is relatively new and has been studied mainly in the context of acute respiratory distress syndrome, since calculated MP includes all ventilator-related variables (e.g. respiratory rate, tidal volume, inspiratory pressure) that, if inexpertly applied, may result in ventilator-induced lung injury [19]. In a previous observational study in prolonged mechanically ventilated, tracheotomized patients, $\mathrm{LTC}_{\mathrm{dyn}}-\mathrm{MP}$ was independently associated with weaning outcome [7], most probably because it agrees well with the pathophysiological basis of prolonged weaning, which is mainly perceived as an imbalance between load (equal to the MP generated by respiratory muscles necessary to provide adequate gas exchange) and capacity of the respiratory pump [5-6].

Specific MP, given it considers lung volume, adjusts for age, gender, and body height [20]. Furthermore, $\mathrm{LTC}_{\mathrm{dyn}}$-MP accounts for changes in airway resistance and chest wall elastance, both of which substantially contribute to the total energy required to inflate the lungs. From a physical perspective, $\mathrm{LTC}_{\mathrm{dyn}}$-MP represents a pressuretime quotient (referred to as "stress intensity") describing the relationship between respiratory rate, pressure and

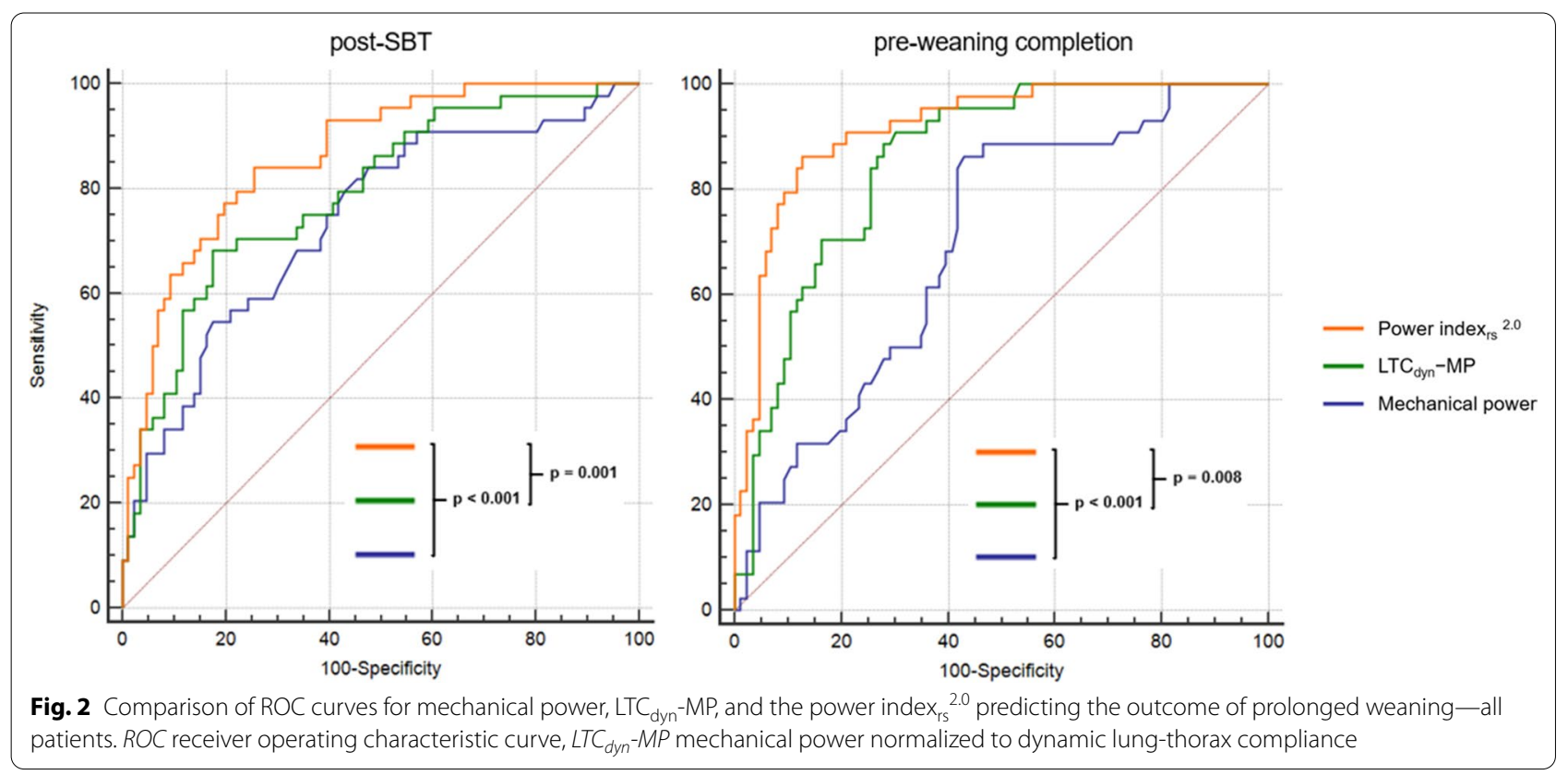


power. Increasing respiratory rate leads to a linear rise in energy transfer, while an increase in pressure (concomitantly increasing tidal volume) results in an exponential increment in power [19].

The formula also reveals the effects of a change in PEEP on the applied specific power in the pressurecontrolled ventilation mode. Provided all other variables remain constant, an increase in PEEP (accompanied by a decrease in driving pressure) results in a reduction in the specific power. Even if an increase in PEEP would lead to recruitment of lung tissue (accompanied by an increase in tidal volume), absolute MP might increase but specific power remains constant, since ventilated lung volume increases to the same extent (reflected by the change in respiratory system compliance). If PEEP reaches the preset inspiratory pressure (driving pressure equals zero), there is no more power transfer from the ventilator to the respiratory system.

The $\mathrm{PI}_{\mathrm{rs}}$ further normalizes applied specific MP to the associated $\mathrm{P}_{\mathrm{a}} \mathrm{CO}_{2}$ (a surrogate for alveolar ventilation), simulating isocapnic conditions and thereby resulting in improved accuracy in predicting the outcome of prolonged weaning. However, this only approximates respiratory physiology, since an increase in pressure (despite increasing tidal volume) will probably not decrease $\mathrm{P}_{\mathrm{a}} \mathrm{CO}_{2}$ when applying an extremely high inspiratory pressure above the upper inflection point of the respiratory system pressure-volume curve, resulting in increased dead space ventilation due to regional lung over-distension [21]. Moreover, $\mathrm{PI}_{\mathrm{rs}}$ does not account for another phenomenon called pendelluft, which may occur during assisted ventilation with low pressure support, leading to increased inspiratory effort with pendular movement of gas between different lung regions, thereby decreasing $\mathrm{CO}_{2}$ removal [22]. Nevertheless, our results suggest that $\mathrm{PI}_{\mathrm{rs}}$ is sufficiently accurate in estimating the MP generated either by the ventilator or by respiratory muscles necessary to provide adequate gas exchange, with the observed increase in diagnostic accuracy of MP indices over the course of weaning most probably explained by our therapeutic interventions, which aimed at improving respiratory mechanics and pulmonary gas exchange, leading to a fall of respiratory muscle workload below the individual fatigue threshold in successfully weaned patients. The observed correlation between specific MP and daily duration of prescribed domiciliary ventilation, which was necessary to sufficiently unload the respiratory pump, further indicates the utility of these indices in estimating respiratory muscle workload during self-ventilation.

Several predictors of weaning and extubation outcome have previously displayed substantial differences in diagnostic accuracy in short-term mechanically ventilated patients [4]. In general, these parameters are determined after a short period of spontaneous breathing (a few minutes after the beginning or at the end of a weaning trial) with or without minimal ventilator assistance. Several of these parameters showed only poor to moderate diagnostic performance in predicting weaning outcome (e.g. maximum inspiratory pressure [23], respiratory rate, tidal volume, or oxygenation [24]). Several attempts have been made to increase accuracy by combining individual parameters into integrative indices. Examples are the CROP [23, 24], CORE [26], Weaning [27], and Integrative weaning [28] indices. CROP and CORE unify respiratory system compliance, (maximum) inspiratory pressure, tidal volume, oxygenation, and respiratory rate, thereby significantly increasing discriminatory performance in predicting the outcome of a weaning trial $[25,26]$. However, assessment of some of these variables is complex (depending on patient's respiratory drive), and none of these indices account for the exponential relationship between pressure and power, and none have been validated in prolonged mechanically ventilated patients. In contrast, mechanical power indices $\left(\mathrm{LTC}_{\mathrm{dyn}}-\mathrm{MP}\right.$ and $\left.\mathrm{PI}_{\mathrm{rs}}\right)$ derive from a physical equation, have a sound pathophysiological rationale, and can be easily assessed during mechanical ventilation without disconnecting the patient from the ventilator.

$\mathrm{PI}_{\mathrm{rs}}$ approximates the load imposed on respiratory muscles during spontaneous breathing. However, since development of hypercapnic respiratory failure is determined by an imbalance between work of breathing and respiratory pump capacity $[5,6]$, one also has to consider the latter when judging a patient's ability to wean from prolonged ventilation. Several studies have assessed trajectories of respiratory muscle function in patients in intensive care, and the impact of this trajectory on mortality and ventilator weaning. While development of, and risk factors for, ventilator-induced diaphragmatic weakness are well described [29-31], little is known about potential reversibility. In a physiological study, Grassi and co-workers demonstrated a correlation between mode of ventilation and changes in diaphragmatic thickness [32]. After early thinning during a short period of controlled ventilation, thickness and thickening fraction recuperated in about $50 \%$ of patients within one week after switching to assisted ventilation. Moreover, Jubran and colleagues showed that respiratory strength (assessed by maximum inspiratory pressure) was well maintained in prolonged mechanically ventilated, tracheotomized patients, and did not significantly change between hospital admission and discharge. They concluded that an increase in muscle strength was not the major determinant of ventilator detachment, but that reduced strength may be a factor in weaning failure [1]. Both studies indicate that in most 
patients respiratory muscle strength recovers early in the course of weaning (e.g. from the moment of switching to assisted ventilation), with weaning outcome mainly determined by the load imposed during spontaneous breathing, consistent with the present results. Nevertheless, prevalence and magnitude of respiratory muscle weakness related to ventilator-associated diaphragmatic dysfunction, bilateral phrenic nerve injury, or pre-existing neuromuscular disease significantly impact the individual fatigue threshold above which ventilatory failure occurs, consequently determining the diagnostic accuracy of MP indices in predicting weaning outcome.

Our study has several strengths. The broad range of medical and surgical patients included increases the applicability of the results. Threshold values and their (internal) validation were assessed prospectively, with the cross-validation technique providing a more thorough estimation of diagnostic performance and generalizability by reducing selection bias (compared to allocating patients to groups based on the time they entered the study). Both the index tests and the reference standard $\left(\mathrm{P}_{\mathrm{a}} \mathrm{CO}_{2}\right.$ during spontaneous breathing) are objective criteria, further reducing the likelihood of bias when determining diagnostic accuracy in predicting weaning outcome. However, this study also has some limitations. Since this was a single-center analysis, external validity is uncertain, despite the broad range of patients we recruited. In addition, MP was calculated using a surrogate formula, proposed for pressure-controlled ventilation [12]. However, in a recent study this simplified, surrogate formula, working under the assumption of a perfectly squared delivered pressure wave, performed better than a more comprehensive formula, without the need for complex calculations [13]. Furthermore, 50.5\% of all variables analyzed were recorded during assistedventilation. Since in a single patient the same inspiratory pressure and PEEP was applied during both a controlled and an assisted breath (in the assisted-controlled mode), leading to identical transrespiratory pressures, absolute MP simply depended on the resulting minute ventilation. In contrast, at a given inspiratory and expiratory pressure, $\mathrm{LTC}_{\mathrm{dyn}}$-MP does not account for changes in minute ventilation when increasing tidal volume due to activation of the diaphragm on assisted-ventilation, potentially leading to a distortion of $\mathrm{P}_{\mathrm{a}} \mathrm{CO}_{2}$ and consequently $\mathrm{PI}_{\mathrm{rs}}$.

\section{Conclusions}

In conclusion, mechanical power normalized to dynamic lung thorax compliance, a surrogate for the applied power per unit of ventilated lung volume, may serve as an estimate of the workload imposed on respiratory muscles during spontaneous breathing, thereby predicting patients' ability to wean from prolonged tracheostomy ventilation. Future changes in clinical practice of tracheostomy weaning could involve weaning protocols including this new predictor in order to help clinicians in guiding the weaning process (e.g. to control the degree of daily extension of spontaneous breathing), with the aim of reducing weaning duration. Further studies are needed to confirm our results.

\section{Abbreviations}

aBGA: Arterial blood gas analysis; ACC: Accuracy; APACHE-II: Acute Physiology and Chronic Health Evaluation II score; AUROC: Area under the receiver operating characteristic curve; BMI: Body mass index; COPD: Chronic obstructive pulmonary disease; DOR: Diagnostic odds ratio; ECLA: Extracorporeal lung assistance; $F_{1}: F_{1}$ score; $F_{i} \mathrm{O}_{2}$ : Fraction of inspired oxygen; HMV-IMV: Home mechanical ventilation-invasive mechanical ventilation; HMV-NIV: Home mechanical ventilation-non-invasive mechanical ventilation; IMV: Invasive mechanical ventilation; LOS: Length of stay; LTC $_{\text {dyn: }}$ : Dynamic lung-thorax compliance; $\mathrm{LTC}_{\mathrm{dyn}}-\mathrm{MP}$ : MP normalized to dynamic lung-thorax compliance; LTOT: Long-term oxygen therapy; MCC: Matthews correlation coefficient; MP: Mechanical power; MV: Mechanical ventilation; NLR: Negative likelihood ratio; No.: Number; NPV: Negative predictive value; PBW-MP: MP normalized to predicted body weight; $\mathrm{PI}_{\mathrm{rs}}$ : Power index of the respiratory system; PLR: Positive likelihood ratio; PPV: Positive predictive value; ROC: Receiver operating characteristic (curve); SB: Spontaneous breathing; SBT: Spontaneous breathing trial; Sens: Sensitivity; Spec: Specificity; VR: Ventilatory ratio.

\section{Supplementary Information}

The online version contains supplementary material available at https://doi. org/10.1186/s12890-021-01566-8.

Additional file 1: Figure S1, Statistical methods-ROC curve analysis and prospective 2-times repeated, twofold cross validation; Figure S2, $2 \times 2$ confusion matrix-Metrics of diagnostic accuracy; Table S1, Clinical characteristics on admission to the weaning center-comparison of groups A/B and C/D; Table S2, Results of prolonged weaning-comparison of patients with weaning success and weaning failure; Table S3, Cross-validated performance of variables derived from the mechanical power analyzed to predict weaning outcome-mean values derived from the test sets; Table S4, Ventilator variables and mechanical power indices predicting the outcome of prolonged weaning — comparison of patients with weaning success and weaning failure; Figure S3, ROC curves for selected variables and mechanical power indices derived from the whole study population $48 \mathrm{~h}$ before weaning completion (PDF 1.0 MB).

\section{Acknowledgements}

Not applicable.

\section{Authors' contributions}

Study concept and design: AG. Acquisition of data: AG, JP, SW. Analysis and interpretation of data: AG, JP, SW, CN. First draft of the manuscript: AG. Critical revision of the manuscript for important intellectual content: AG, JP, SW, CN. Statistical analysis: AG. All authors have read and approved the final version of the manuscript.

Funding

None.

Availability of data and materials

The datasets used and/or analyzed during the current study are available from the corresponding author on reasonable request. 


\section{Declarations}

\section{Ethics approval and consent to participate}

The study was approved by the local institutional review board for human studies (Ethics Committee of the State Chamber of Physicians of BadenWuerttemberg, Germany, File Number F-2018-116) and performed in accordance with the Declaration of Helsinki. Written informed consent was obtained from all patients or a legal representative.

\section{Consent for publication}

Not applicable.

\section{Competing interests}

The authors declare that they have no competing interests.

\section{Author details}

'Department of Pulmonology and Respiratory Medicine, Schillerhoehe Lung Clinic (affiliated to the Robert-Bosch-Hospital GmbH, Stuttgart), Solitudestrasse 18, 70839 Gerlingen, Germany. ${ }^{2}$ German Center for Lung Research (DZL), München, Germany.

Received: 9 December 2020 Accepted: 21 May 2021 Published online: 17 June 2021

\section{References}

1. Jubran A, Grant BJB, Duffner LA, et al. Long-term outcome after prolonged mechanical ventilation. Am J Respir Crit Care Med. 2019;199(12):1508-16.

2. Bonnici DM, Sanctuary T, Warren A, et al. Prospective observational cohort study of patients with weaning failure admitted to a specialist weaning, rehabilitation and home mechanical ventilation centre. BMJ Open. 2016;6:e010025

3. Ghiani A, Sainis A, Sainis G, Neurohr C. Anemia and red blood cell transfusion practice in prolonged mechanically ventilated patients admitted to a specialized weaning center: an observational study. BMC Pulm Med. 2019;19:250.

4. Baptistella AF, Sarmento FJ, Ribeiro da Silva K, et al. Predictive factors of weaning from mechanical ventilation and extubation outcome: a systematic review. J Crit Care. 2018;48:56-62.

5. Purro A, Appendini L, De Gaetano A, Gudjonsdottir M, Donner CF, Rossi A. Physiologic determinants of ventilator dependence in longterm mechanically ventilated patients. Am J Respir Crit Care Med. 2000;161(4):1115-23.

6. Carlucci A, Ceriana P, Prinianakis G, Fanfulla F, Colombo R, Nava S. Determinants of weaning success in patients with prolonged mechanical ventilation. Crit Care. 2009:13:R97.

7. Ghiani A, Paderewska J, Sainis A, Crispin A, Walcher S, Neurohr C. Variables predicting weaning outcome in prolonged mechanically ventilated tracheotomized patients: a retrospective study. J Intensive Care. 2020;8:19.

8. Boles JM, Bion J, Connors A, et al. Weaning from mechanical ventilation. Eur Respir J. 2007;29(5):1033-56.

9. Schönhofer B, Geiseler J, Dellweg D, et al. Prolonged weaning: S2kguideline published by the German Respiratory Society. Pneumologie. 2019;73(12):723-814.

10. Jubran A, Grant BJ, Duffner LA, et al. Effect of pressure support vs unassisted breathing through a tracheostomy collar on weaning duration in patients requiring prolonged mechanical ventilation: a randomized trial. JAMA. 2013;309(7):671-7.

11. Okabe Y, Asaga T, Bekku S, et al. Lung-thorax compliance measured during a spontaneous breathing trial is a good index of extubation failure in the surgical intensive care unit: a retrospective cohort study. J Intensive Care. 2018:6:44
12. Becher T, van der Staay M, Schädler D, Frerichs I, Weiler N. Calculation of mechanical power for pressure-controlled ventilation. Intensive Care Med. 2019:45(9):1321-3.

13. Chiumello D, Gotti M, Guanziroli M, et al. Bedside calculation of mechanical power during volume- and pressure controlled mechanical ventilation. Crit Care. 2020;24:417.

14. Sinha P, Fauvel NJ, Singh P, Soni N. Analysis of ventilatory ratio as a novel method to monitor ventilatory adequacy at the bedside. Crit Care. 2013;17:R34.

15. Brower RG, Matthay MA, Morris A, Schoenfeld D, Thompson BT, Wheeler A. Ventilation with lower tidal volumes as compared with traditional tidal volumes for acute lung injury and the acute respiratory distress syndrome. N Engl J Med. 2000;342(18):1301-8.

16. Windisch W, Dreher M, Geiseler J, et al. Guidelines for non-invasive and invasive home mechanical ventilation for treatment of chronic respiratory failure: update 2017. Pneumologie. 2017;71(11):722-95.

17. Arlot S, Celisse A. A survey of cross-validation procedures for model selection. Stat Surv. 2010;4:40-79.

18. Coppola S, Caccioppola A, Froio S, et al. Effect of mechanical power on intensive care mortality in ARDS patients. Crit Care. 2020;24:246.

19. Gattinoni L, Tonetti T, Cressoni M, et al. Ventilator-related causes of lung injury: the mechanical power. Intensive Care Med. 2016;42(10):1567-75.

20. Quanjer PH, Stanojevic S, Cole TJ, et al. Multi-ethnic reference values for spirometry for the 3-95-yr age range: the global lung function 2012 equations. Eur Respir J. 2012;40(6):1324-43.

21. Blanch L, Lopez-Aguilar J, Lucangelo U. Dead space in acute respiratory distress syndrome: more than a feeling! Crit Care. 2016:20:214.

22. Coppadoro A, Grassi A, Giovannoni C, et al. Occurrence of pendelluft under pressure support ventilation in patients who failed a spontaneous breathing trial: an observational study. Ann Intensive Care. 2020;10:39.

23. Wu YK, Kao KC, Hsu KH, Hsieh MJ, Tsai YH. Predictors of successful weaning from prolonged mechanical ventilation in Taiwan. Respir Med. 2009;103(8):1189-95.

24. Savi A, Teixeira C, Silva JM, et al. Weaning predictors do not predict extubation failure in simple-to-wean patients. J Crit Care. 2012;27(221):e1-8.

25. Yang KL, Tobin MJ. A prospective study of indexes predicting the outcome of trials of weaning from mechanical ventilation. N Engl J Med. 1991:324(21):1445-50.

26. Delisle S, Francoeur M, Albert M, Ouellet P, Bellemare P, Arsenault P. Preliminary evaluation of a new index to predict the outcome of a spontaneous breathing trial. Respir Care. 2011;56(10):1500-5.

27. Huaringa AJ, Wang A, Haro MH, Leyva FJ. The weaning index as a predictor of weaning success. J Intensive Care Med. 2012;28(6):369-74.

28. Nemer SN, Barbas CSV, Caldeira JB, et al. A new integrative weaning index of discontinuation from mechanical ventilation. Crit Care. 2009;13:R152.

29. Levine S, Nguyen T, Taylor N, et al. Rapid disuse atrophy of diaphragm fibers in mechanically ventilated humans. N Eng J Med. 2008;358(13):1327-35.

30. Dres M, Dube BP, Mayaux J, et al. Coexistance and impact of limb muscle and diaphragm weakness at time of liberation from mechanical ventilation in medical intensive care unit patients. Am J Respir Crit Care Med. 2017;195(1):57-66.

31. Goligher EC, Dres M, Fan E, et al. Mechanical ventilation-induced diaphragm atrophy strongly impacts clinical outcomes. Am J Respir Crit Care Med. 2018;197(2):204-13.

32. Grassi A, Ferlicca D, Lupieri E, et al. Assisted mechanical ventilation promotes recovery of diaphragmatic thickness in critically ill patients: a prospective observational study. Crit Care. 2020;24:85.

\section{Publisher's Note}

Springer Nature remains neutral with regard to jurisdictional claims in published maps and institutional affiliations. 